Research Article

\title{
Some Properties and Distribution of the Zeros of the $q$-Sigmoid Polynomials
}

\author{
Jung Yoog Kang $\mathbb{D}$ \\ Department of Mathematics Education, Silla University, Busan, Republic of Korea \\ Correspondence should be addressed to Jung Yoog Kang; jykang@silla.ac.kr
}

Received 13 October 2019; Revised 4 May 2020; Accepted 8 June 2020; Published 3 July 2020

Academic Editor: Piergiulio Tempesta

Copyright (c) 2020 Jung Yoog Kang. This is an open access article distributed under the Creative Commons Attribution License, which permits unrestricted use, distribution, and reproduction in any medium, provided the original work is properly cited.

The sigmoid polynomials combining $q$-numbers and various properties of their polynomials are thoroughly presented in this paper. Based on several properties of $q$-numbers, we derive some identities of $q$-sigmoid polynomials. Also, from explicit polynomials, we can observe structures of the zeros of the sigmoid polynomials which are related to $q$-numbers.

\section{Introduction}

For any $n \in \mathbb{C}$, the $q$-number can be defined as follows:

$$
[n]_{q}=\frac{1-q^{n}}{1-q}=\sum_{0 \leq i \leq n-1} q^{i}=1+q+q^{2}+\cdots+q^{n-1} .
$$

The discovery of $q$-numbers by Jackson has been applied to various fields of mathematics such as $q$-differential equations, $q$-integrals and differentials, and $q$-series, and many properties have been studied by mathematicians (see [1-10]). It is also applied to polynomials, and their properties are studied in various ways in combination with Bernoulli, Euler, and Genocchi polynomials, which are considered important. As a start, we would like to introduce several definitions related to $q$-numbers used in this paper.

Definition 1. The Gaussian binomial coefficients are defined by

$$
\left(\begin{array}{c}
m \\
r
\end{array}\right)_{q}=\left[\begin{array}{l}
m \\
r
\end{array}\right]_{q}= \begin{cases}0, & \text { if } r>m, \\
\frac{\left(1-q^{m}\right)\left(1-q^{m-1}\right) \ldots\left(1-q^{m-r+1}\right)}{(1-q)\left(1-q^{2}\right) \ldots\left(1-q^{r}\right)}, & \text { if } r \leq m,\end{cases}
$$

where $m$ and $r$ are non-negative integers. For $r=0$, the value is 1 since the numerator and the denominator are both empty products. Like the classical binomial coefficients, the Gaussian binomial coefficients are center-symmetric. There are analogues of the binomial formula, and this definition has a number of properties (see [1-25]).

Theorem 1. Let $n, k$ be non-negative integers. Then, we get

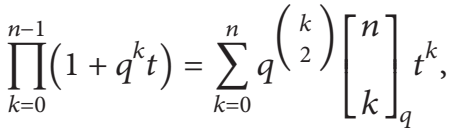

$$
\begin{aligned}
& \prod_{k=0}^{n-1} \frac{1}{\left(1-q^{k} t\right)}=\sum_{k=0}^{\infty}\left[\begin{array}{c}
n+k-1 \\
k
\end{array}\right]_{q} t^{k}
\end{aligned}
$$


Definition 2. Let $z$ be any complex number with $|z|<1$. Two forms of $q$-exponential functions are expressed as

$$
\begin{aligned}
e_{q}(z) & =\sum_{n=0}^{\infty} \frac{z^{n}}{[n]_{q} !} \\
e_{q^{-1}}(z) & =\sum_{n=0}^{\infty} \frac{z^{n}}{[n]_{q^{-1}} !}=\sum_{n=0}^{\infty} q\left(\begin{array}{c}
n \\
2
\end{array}\right) \frac{z^{n}}{[n]_{q} !}
\end{aligned}
$$

Definition 3. The definition of the q-derivative operator of any function $f$ follows that

$$
D_{q} f(x)=\frac{f(x)-f(q x)}{(1-q) x}, \quad x \neq 0,
$$

and $D_{q} f(0)=f^{\prime}(0)$.

We can prove that $f$ is differentiable at 0 , and it is clear that $D_{q} x^{n}=[n]_{q} x^{n-1}$.

Definition 4. We define the $q$-integral as

$$
\int_{0}^{b} f(x) d_{q} x=(1-q) b \sum_{j=0}^{\infty} q^{j} f\left(q^{j} b\right) .
$$

If this function, $f(x)$, is differentiable on the point $x$, the $q$-derivative in Definition 3 goes to the ordinary derivative in the classical analysis when $q \longrightarrow 1$.

In a deep learning network, we pass the nonlinear function through the nonlinear function, rather than passing it directly to the next layer. The function used at this time is called the activation function. Among these activation functions, there is a sigmoid function. The definition of sigmoid function is as follows.

Definition 5. Let $z \in \mathbb{C}$. Then, the sigmoid function is expressed as

$$
s(z)=\frac{1}{1+e^{-z}} .
$$

In order to find various applications, various studies were done by investigating the sigmoid function. For example, a variant sigmoid function with three parameters has been employed in order to explain hybrid sigmoidal networks, and sigmoid function, which is also called logistic function, has been defined using flexible sigmoidal mixed models based on logistic family curves for medical applications (see [15-19,23]). The following theorem has several basic properties for sigmoid polynomials.

Theorem 2. Let $x \in \mathbb{C}$. Then, the following holds:

$$
\begin{aligned}
S_{n}(x) & =\sum_{k=0}^{n}\left(\begin{array}{l}
n \\
k
\end{array}\right) S_{k} x^{n-k}, \\
S_{n}(x+y) & =\sum_{k=0}^{n}\left(\begin{array}{l}
n \\
k
\end{array}\right) S_{k}(x) y^{n-k}, \\
S_{n}+\sum_{k=0}^{n}\left(\begin{array}{l}
n \\
k
\end{array}\right)(-1)^{k} S_{n-k} & = \begin{cases}1, & \text { if } n=0, \\
0, & \text { if } n \neq 0, \\
(-1)^{n} S_{n}(-1-x) & =S_{n}(x) .\end{cases}
\end{aligned}
$$

In this paper, in order to confirm the relationship between the sigmoid polynomial including $q$-numbers and other polynomials, let us check the following famous polynomials including $q$-numbers.

The Bernoulli, Euler, and Genocchi polynomials have been widely studied in various mathematical applications including number theory, finite difference calculus, combinatorial analysis, and $p$-adic analytic number theory. These numbers and polynomials are very well known and most mathematicians are familiar with them because of their importance (see $[1,11,13,20,22,24,25])$.

Definition 6. $q$ - Bernoulli numbers, $B_{n, q}$, and polynomials, $B_{n, q}(x)$, can be expressed as

$$
\begin{gathered}
\sum_{n=0}^{\infty} B_{n, q} \frac{t^{n}}{n !}=\frac{t}{e_{q}(t)-1}, \\
\sum_{n=0}^{\infty} B_{n, q}(x) \frac{t^{n}}{n !}=\frac{t}{e_{q}(t)-1} e_{q}(t x),
\end{gathered}
$$

respectively; $q$-Euler numbers, $E_{n, q}$, and polynomials, $E_{n, q}(x)$, are expressed as follows:

$$
\begin{aligned}
\sum_{n=0}^{\infty} E_{n, q} \frac{t^{n}}{n !} & =\frac{[2]_{q}}{e_{q}(t)+1}, \\
\sum_{n=0}^{\infty} E_{n, q}(x) \frac{t^{n}}{n !} & =\frac{[2]_{q}}{e_{q}(t)+1} e_{q}(t x),
\end{aligned}
$$

and finally, $q$-Genocchi numbers, $G_{n, q}$, and polynomials, $G_{n, q}(x)$, respectively, are expressed as follows:

$$
\begin{aligned}
\sum_{n=0}^{\infty} G_{n} \frac{t^{n}}{n !} & =\frac{[2]_{q} t}{e_{q}(t)+1}, \\
\sum_{n=0}^{\infty} G_{n}(x) \frac{t^{n}}{n !} & =\frac{[2]_{q} t}{e_{q}(t)+1} e_{q}(t x),
\end{aligned}
$$

Finding the properties of sigmoid polynomials combined with $q$-numbers in various ways is the main topic of this paper. In Section 2, we look for the basic properties of $q$-sigmoid polynomials and find relationships with Bernoulli, Euler, and Genocchi polynomials that can cope with this polynomial. We also check the relationship between the numbers and the polynomials and symmetry and find the identities using $q$-differential equations. In Section 3, approximate roots are sought based on the properties described in Section 2. We will observe the changes of the approximate roots of $q$-sigmoid polynomials according to the change of $q$-number and confirm some assumptions based on it.

\section{Some Basic Properties of Sigmoid Polynomials Combining $q$-Numbers}

In this section, we first define the number of sigmoid and polynomials that combine the $q$-numbers, plus the polynomials associated with the two variables. We find the 
properties and identities of polynomials based on their definition and find relationships with other polynomials. We also use the $q$-number differential formula to find the identities of the polynomials for two parameters.

Definition 7. Let $0<q<1$ and $q \in \mathbb{R}$. Then, we define $q$-sigmoid polynomials as

$$
\sum_{n=0}^{\infty} \mathcal{S}_{n, q}(x) \frac{t^{n}}{[n]_{q} !}=\frac{1}{e_{q}(-t)+1} e_{q}(t x) .
$$

From Definition 7, we have

$$
\sum_{n=0}^{\infty} \mathcal{S}_{n, q}(0) \frac{t^{n}}{[n]_{q} !}=\frac{1}{e_{q}(-t)+1}=\sum_{n=0}^{\infty} \mathcal{S}_{n, q} \frac{t^{n}}{[n]_{q} !},
$$

where we call $\mathcal{S}_{n, q}$ as $q$-sigmoid numbers. Also, we can consider $q$-sigmoid polynomials of two parameters as

$$
\sum_{n=0}^{\infty} \mathcal{S}_{n, q}(x, y) \frac{t^{n}}{[n]_{q} !}=\frac{1}{e_{q}(-t)+1} e_{q}(t x) e_{q}(t y) .
$$

We note that $q$-sigmoid polynomials are the same as sigmoid polynomials when $q$ approaches 1 .

Theorem 3. For $0<q<1$ and $x \in \mathbb{C}$, we have

$$
\mathcal{S}_{n, q}(x)=\sum_{k=0}^{n}\left[\begin{array}{l}
n \\
k
\end{array}\right]_{q} \mathcal{S}_{k, q} x^{n-k} .
$$

Proof. From Definition 7, we can find a relation between $q$-sigmoid numbers and polynomials such as

$$
\sum_{n=0}^{\infty} \mathcal{S}_{n, q}(x) \frac{t^{n}}{[n]_{q} !}=\frac{1}{e_{q}(-t)+1} e_{q}(t x)=\sum_{n=0}^{\infty} \mathcal{S}_{n, q} \frac{t^{n}}{[n]_{q} !} \sum_{n=0}^{\infty} x^{n} \frac{t^{n}}{[n]_{q} !}=\sum_{n=0}^{\infty}\left(\sum_{k=0}^{n}\left[\begin{array}{l}
n \\
k]_{q}
\end{array} \mathcal{S}_{k, q} x^{n-k}\right) \frac{t^{n}}{[n]_{q} !}\right.
$$

Comparing the both sides of $t^{n} /[n]_{q}$, the required relation follows immediately.

Corollary 1. From Theorem 3, the following holds:

$$
\begin{aligned}
\mathcal{S}_{n, q}(x+y) & =\sum_{k=0}^{n}\left[\begin{array}{l}
n \\
k
\end{array}\right]_{q} S_{k, q}(x+y)^{n-k}, \\
\mathcal{S}_{n, q}(x, y) & =\sum_{k=0}^{n}\left[\begin{array}{l}
n \\
k
\end{array}\right]_{q} \mathcal{S}_{k, q}(x) y^{n-k}, \\
\mathcal{S}_{n, q}(x, y) & =\sum_{l=0}^{n} \sum_{k=0}^{n-l}\left[\begin{array}{l}
n \\
l
\end{array}\right]_{q}\left[\begin{array}{c}
n-l \\
k
\end{array}\right]_{q} \mathcal{S}_{l, q} x^{n-l-k} y^{k} .
\end{aligned}
$$

Theorem 4. Let $n$ be a non-negative integer. Then, we find

$$
x^{n}=\sum_{k=0}^{n}\left[\begin{array}{l}
n \\
k
\end{array}\right]_{q}(-1)^{n-k} \mathcal{S}_{k, q}(x)+\mathcal{S}_{n, q}(x) .
$$

Proof. Considering $e_{q}(-t)+1 \neq 0$ in Definition 7 , we can express

$$
\sum_{n=0}^{\infty} \mathcal{S}_{n, q}(x) \frac{t^{n}}{[n]_{q} !}\left(e_{q}(-t)+1\right)=\sum_{n=0}^{\infty} x^{n} \frac{t^{n}}{[n]_{q} !} .
$$

Using $q$-exponential function in the equation above, we can find

$$
\sum_{n=0}^{\infty}\left(\sum_{k=0}^{n}\left[\begin{array}{l}
n \\
k
\end{array}\right]_{q}(-1)^{n-k} \mathcal{S}_{k, q}(x)+\mathcal{S}_{n, q}(x)\right) \frac{t^{n}}{[n]_{q} !}=\sum_{n=0}^{\infty} x^{n} \frac{t^{n}}{[n]_{q} !}
$$

which gives the required result.
Corollary 2. From Theorem 4, one obtains

$$
\sum_{k=0}^{n}\left[\begin{array}{l}
n \\
k
\end{array}\right]_{q}(-1)^{n-k} \mathcal{S}_{k, q}+\mathcal{S}_{n, q}= \begin{cases}1, & \text { if } n=0 \\
0, & \text { if } n \neq 0\end{cases}
$$

Theorem 5. Let $0<q^{-1}<1$ and $n$ be a non-negative integer. Then, we derive

$$
\begin{aligned}
& \sum_{k=0}^{n}\left[\begin{array}{l}
n \\
k
\end{array}\right]_{q}(-1)^{k} q^{(k / 2)+(n-k / 2)} \mathcal{S}_{n-k, q^{-1}}(x)+q^{(n / 2)} \mathcal{S}_{n, q^{-1}}(x) \\
& \quad=q^{(n / 2)} x^{n}, \\
& \sum_{k=0}^{n}\left[\begin{array}{l}
n \\
k
\end{array}\right]_{q}(-1)^{k} q^{(k / 2)+(n-k / 2)} \mathcal{S}_{n-k, q^{-1}}+q^{(n / 2)} \mathcal{S}_{n, q^{-1}} \\
& \quad= \begin{cases}1, & \text { if } n=0 \\
0, & \text { if } n \neq 0 .\end{cases}
\end{aligned}
$$

Proof

(i) For $e_{q^{-1}}(t) \neq-1$ in Definition 7 , we can have

$$
\sum_{n=0}^{\infty} \mathcal{S}_{n, q^{-1}}(x) \frac{t^{n}}{[n]_{q^{-1}} !}\left(e_{q^{-1}}(-t)+1\right)=e_{q^{-1}}(t x)
$$

And we note that $[n]_{q^{-1}} !=q^{-\left(\begin{array}{l}n \\ 2\end{array}\right)}[n]_{q}$ !. Applying this property in the equation above, we can find

$$
\begin{aligned}
& \sum_{n=0}^{\infty} q^{(n / 2)} \mathcal{S}_{n, q^{-} 1}(x) \frac{t^{n}}{[n]_{q} !}\left(\sum_{n=0}^{\infty}(-1)^{n} q^{(n / 2)} \frac{t^{n}}{[n]_{q} !}+1\right) \\
& =\sum_{n=0}^{\infty} q^{(n / 2)} x^{n} \frac{t^{n}}{[n]_{q} !}
\end{aligned}
$$


Using Cauchy's product, we obtain

$$
\sum_{n=0}^{\infty}\left(\sum_{k=0}^{n}\left[\begin{array}{l}
n \\
k
\end{array}\right]_{q}(-1)^{k} q^{(k / 2)+(n-k / 2)} \mathcal{S}_{n-k, q^{-} 1}(x)+q^{(n / 2)} \mathcal{S}_{n, q^{-1}}(x)\right) \frac{t^{n}}{[n]_{q} !}=\sum_{n=0}^{\infty} q^{(n / 2)} x^{n} \frac{t^{n}}{[n]_{q} !}
$$

which gives the required result.

(ii) We omit a proof of (ii) due to its similarity to (i).

Theorem 6. Let $a, b$ be any non-negative integers. Then, we find symmetric property as

$$
\sum_{k=0}^{n}\left[\begin{array}{l}
n \\
k
\end{array}\right]_{q} \frac{\mathcal{S}_{n-k, q}(a x) \mathcal{S}_{k, q}(b y)}{a^{n-k} b^{k}}=\sum_{k=0}^{n}\left[\begin{array}{l}
n \\
k
\end{array}\right]_{q} \frac{\mathcal{S}_{n-k, q}(b x) \mathcal{S}_{k, q}(a y)}{a^{k} b^{n-k}}
$$

Proof. We can consider that

$$
A:=\frac{e_{q}(t x) e_{q}(t y)}{\left(e_{q}(-(t / a))+1\right)\left(e_{q}(-(t / b))+1\right)} \text {. }
$$

From the form $A$, we can find

$$
\begin{aligned}
A & =\sum_{n=0}^{\infty} \frac{1}{a^{n}} \mathcal{S}_{n, q}(a x) \frac{t^{n}}{[n]_{q} !} \sum_{n=0}^{\infty} \frac{1}{b^{n}} \mathcal{S}_{n, q}(b y) \frac{t^{n}}{[n]_{q} !} \\
& =\sum_{n=0}^{\infty}\left(\sum_{k=0}^{n}\left[\begin{array}{l}
n \\
k
\end{array}\right]_{q} \frac{1}{a^{n-k} b^{k}} \mathcal{S}_{n-k, q}(a x) \mathcal{S}_{k, q}(b y)\right) \frac{t^{n}}{[n]_{q} !}
\end{aligned}
$$

or equivalently

$$
A=\sum_{n=0}^{\infty}\left(\sum_{k=0}^{n}\left[\begin{array}{l}
n \\
k
\end{array}\right]_{q} \frac{1}{b^{n-k} a^{k}} \mathcal{S}_{n-k, q}(b x) \mathcal{S}_{k, q}(a y)\right) \frac{t^{n}}{[n]_{q} !}
$$

The required relation follows on comparing the coefficients of $t^{n} /[n]_{q}$ ! in both sides.

Corollary 3. Let $s, t$ be any non-negative integers without 0 from Theorem 6; one obtains

$$
\begin{aligned}
\sum_{k=0}^{n}\left[\begin{array}{l}
n \\
k
\end{array}\right]_{q} s^{n-k} t^{k} \mathcal{S}_{n-k, q}\left(\frac{x}{s}\right) \mathcal{S}_{k, q}\left(\frac{y}{t}\right) \\
=\sum_{k=0}^{n}\left[\begin{array}{l}
n \\
k
\end{array}\right]_{q}^{k} t^{n-k} \mathcal{S}_{n-k, q}\left(\frac{x}{t}\right) \mathcal{S}_{k, q}\left(\frac{y}{s}\right) .
\end{aligned}
$$

Theorem 7. For $0<q<1$ and $q \in \mathbb{R}$, we find some relations as

$$
\begin{array}{r}
\mathcal{S}_{n, q}(x, y)=\frac{1}{[2]_{q}} \sum_{l=0}^{n}\left[\begin{array}{l}
n \\
l
\end{array}\right]_{q}\left(\sum_{k=0}^{l}\left[\begin{array}{l}
l \\
k
\end{array}\right]_{q}^{\left.\frac{\mathcal{S}_{l-k, q}(x)}{m^{n-l+k}}+\frac{\mathcal{S}_{l, q}(x)}{m^{n-l}}\right) E_{n-l, q}(m y), \quad \text { where } E_{n, q}(x) \text { is the } q-\text { Euler polynomial, }}\right. \\
{[n]_{q} \mathcal{S}_{n-l, q}(x, y)=m \sum_{l=0}^{n}\left[\begin{array}{l}
n \\
l
\end{array}\right]_{q}\left(\sum_{k=0}^{l}\left[\begin{array}{l}
l \\
k
\end{array}\right]_{q} \frac{\mathcal{S}_{l-k, q}(x)-m^{k} \mathcal{S}_{l, q}(x)}{m^{k+n-l}}\right) B_{n-l, q}(m y), \quad \text { where } B_{n}(x) \text { is the Bernoulli polynomial, }} \\
(1+q)[n]_{q} \mathcal{S}_{n-l, q}(x, y)=\sum_{l=0}^{n}\left[\begin{array}{l}
n \\
l
\end{array}\right]_{q}\left(\sum_{k=0}^{l}\left[\begin{array}{l}
l \\
k
\end{array}\right]_{q}^{\left.\frac{\mathcal{S}_{l-k, q}(x)+m^{k} \mathcal{S}_{l, q}(x)}{m^{k+n-l}}\right)} G_{n-l, q}(m y), \quad \text { where } G_{n}(x)\right. \text { is the Genocchi polynomial. }
\end{array}
$$

Proof (i) We suppose that

$$
B=\frac{e_{q}(t / m) e_{q}(t x) e_{q}(t y)}{\left(e_{q}(-t)+1\right)\left(e_{q}(t / m)+1\right)}+\frac{e_{q}(t x) e_{q}(t y)}{\left(e_{q}(-t)+1\right)\left(e_{q}(t / m)+1\right)} .
$$


From the form $B$, we can obtain

$$
\begin{aligned}
B= & \frac{1}{[2]_{q}} \sum_{n=0}^{\infty}\left(\sum_{k=0}^{n}\left[\begin{array}{l}
n \\
k
\end{array}\right]_{q} \frac{\mathcal{S}_{n-k, q}(x)}{m^{k}}\right) \frac{t^{n}}{[n]_{q} !} \sum_{n=0}^{\infty} \frac{\mathrm{E}_{n, q}(m y)}{m^{n}} \frac{t^{n}}{[n]_{q} !} \\
& +\frac{1}{[2]_{q}} \sum_{n=0}^{\infty}\left(\sum_{l=0}^{n}\left[\begin{array}{l}
n \\
l
\end{array}\right]_{q} \frac{\mathcal{S}_{l, q}(x) \mathrm{E}_{n-l, q}(m y)}{m^{n-l}}\right) \frac{t^{n}}{[n]_{q} !} \\
= & \frac{1}{[2]_{q}} \sum_{n=0}^{\infty}\left(\sum_{l=0}^{n}\left[\begin{array}{l}
n \\
l
\end{array}\right]_{q} \sum_{k=0}^{l}\left[\begin{array}{l}
n \\
k
\end{array}\right]_{q} \frac{\mathcal{S}_{n-k, q}(x) \mathrm{E}_{n-l, q}(m y)}{m^{n-l+k}}\right) \frac{t^{n}}{[n]_{q} !} \\
& +\frac{1}{[2]_{q}} \sum_{n=0}^{\infty}\left(\sum_{l=0}^{n}\left[\begin{array}{l}
n \\
l
\end{array}\right]_{q} \frac{\mathcal{S}_{l, q}(x) \mathrm{E}_{n-l, q}(m y)}{m^{n-l}}\right) \frac{t^{n}}{[n]_{q} !} \\
B= & \sum_{n=0}^{\infty} \mathcal{S}_{n, q}(x, y) \frac{t^{n}}{[n]_{q} !}
\end{aligned}
$$

Therefore, we find result of Theorem 7 (i), (ii), and (iii). To find results of (ii) and (iii), we consider

$$
\begin{aligned}
C & :=\frac{e_{q}(t / m) e_{q}(t x) e_{q}(t y)}{\left(e_{q}(-t)+1\right)\left(e_{q}(t / m)-1\right)}-\frac{e_{q}(t x) e_{q}(t y)}{\left(e_{q}(-t)+1\right)\left(e_{q}(t / m)-1\right)}, \\
D & :=\frac{e_{q}(t / m) e_{q}(t x) e_{q}(t y)}{\left(e_{q}(-t)+1\right)\left(e_{q}(t / m)+1\right)}+\frac{e_{q}(t x) e_{q}(t y)}{\left(e_{q}(-t)+1\right)\left(e_{q}(t / m)+1\right)} .
\end{aligned}
$$

We omit the proof of (ii) and (iii) because we can derive required results in the same method as (i).

Theorem 8. Let $0<q<1, q \in \mathbb{R}$, and $n \in \mathbb{Z}$. $q$-differential of $q$-sigmoid polynomials is derived as

$$
\begin{gathered}
\mathscr{D}_{q, y} \mathcal{S}_{n, q}(x, y)=[n]_{q} \mathcal{S}_{n-1, q}(x, y), \\
\mathscr{D}_{q} \mathcal{S}_{n, q}(x)=[n]_{q} \mathcal{S}_{n-1, q}(x) .
\end{gathered}
$$

Proof

(i) Using $q$-derivative in Corollary 1 (ii), we have

$$
\begin{aligned}
\mathscr{D}_{q, y} \mathcal{S}_{n, q}(x, y) & =\sum_{k_{\bar{n}} 0}^{n}\left[\begin{array}{l}
n \\
k
\end{array}\right]_{q} \mathcal{S}_{k, q}(x) \mathscr{D}_{q, y} y^{n-k} \\
& =\sum_{k=0}^{n}\left[\begin{array}{l}
n \\
k
\end{array}\right]_{q}[n-k]_{q} \mathcal{S}_{k, q}(x) y^{n-k-1} \\
& =[n]_{q} \sum_{k=0}^{n-1}\left[\begin{array}{c}
n-1 \\
k
\end{array}\right]_{q} \mathcal{S}_{k, q}(x) y^{n-k-1} .
\end{aligned}
$$

Applying Corollary 1 (ii) again in the equation above, we complete a proof of (i).

(ii) We omit the proof of (ii) because we can derive required results in the same method as (i) and use Theorem 3.

Theorem 9. For $|q|<1$, we obtain

$$
\begin{aligned}
& \mathcal{S}_{n, q}(x, y)=\sum_{l=0}^{n}\left[\begin{array}{l}
n \\
l
\end{array}\right]_{q}\left(\sum_{k=0}^{l}\left[\begin{array}{l}
l \\
k
\end{array}\right]_{q} q^{\left.\left(\begin{array}{c}
l-k \\
2
\end{array}\right) x^{k} \delta_{l-k, q^{-1}}(-1)\right) y^{n-l}}\right. \\
& \mathcal{S}_{n, q}(x)=\sum_{k=0}^{n}\left[\begin{array}{l}
n \\
k
\end{array}\right]_{q} q^{(k / 2)} \mathcal{S}_{k, q^{-1}}(-1) x^{n-k} \\
& \text { Proof }
\end{aligned}
$$

(i) Transforming the generating function of $q$-sigmoid polynomials, we can have

$$
\begin{aligned}
\sum_{n=0}^{\infty} \mathcal{S}_{n, q}(x, y) \frac{t^{n}}{[n]_{q} !} & =\frac{e_{q^{-1}}(t)}{1+e_{q^{-1}}(t)} e_{q}(t x) e_{q}(t y) \\
& =\sum_{n=0}^{\infty} \mathcal{S}_{n, q^{-1}}(-1) \frac{t^{n}}{[n]_{q^{-1}} !} \sum_{n=0}^{\infty} x^{n} \frac{t^{n}}{[n]_{q} !} \sum_{n=0}^{\infty} y^{n} \frac{t^{n}}{[n]_{q} !}
\end{aligned}
$$

We can note a property of $q$-numbers such as $[n]_{q-1} !=q^{-\left(\begin{array}{l}n \\ 2\end{array}\right)}[n]_{q} !$. Applying the property in the equation above, we have

$$
\sum_{n=0}^{\infty} \mathcal{S}_{n, q}(x, y) \frac{t^{n}}{[n]_{q} !}=\sum_{n=0}^{\infty}\left(\sum_{l=0}^{n}\left[\begin{array}{l}
n \\
l
\end{array}\right]_{q} y^{n-l} \sum_{k=0}^{l}\left[\begin{array}{l}
l \\
k
\end{array}\right]_{q}^{\left(\begin{array}{c}
l-k \\
2
\end{array}\right)} \mathcal{S}_{l-k, q^{-1}}(-1) x^{k}\right) \frac{t^{n}}{[n]_{q} !}
$$

Hence, we can find the required result.

Theorem 10. Let $|q|<1$. Then, we investigate

(ii) We omit a proof of (ii) due to its similarity to (i).

$$
\begin{aligned}
& \sum_{l=0}^{n-1}\left[\begin{array}{c}
n-1 \\
l
\end{array}\right]_{q} \mathcal{S}_{l, q}(x)=\sum_{l=0}^{n-1}\left[\begin{array}{c}
n-1 \\
l
\end{array}\right]_{q} \sum_{k=0}^{l}\left[\begin{array}{l}
l \\
k
\end{array}\right]_{q} q^{\left(\begin{array}{c}
l-k \\
2
\end{array}\right) x^{k} \mathcal{S}_{l-k, q^{-1}}(-1),} \\
& \sum_{k=0}^{n-1}\left[\begin{array}{c}
n-1 \\
k
\end{array}\right]_{q} \mathcal{S}_{k, q}=\sum_{k=0}^{n-1}\left[\begin{array}{c}
n-1 \\
k
\end{array}\right]_{q}^{\left(\begin{array}{l}
k \\
2
\end{array}\right)} \mathcal{S}_{k, q^{-1}}(-1) .
\end{aligned}
$$


Proof

(i) Using q-derivative in Theorem 10, we can find

$$
\begin{aligned}
\mathscr{D}_{q, y} \mathcal{S}_{n, q}(x, y) & =\sum_{l=0}^{n}\left[\begin{array}{l}
n \\
l
\end{array}\right]_{q}\left(\sum_{k=0}^{l}\left[\begin{array}{l}
l \\
k
\end{array}\right]_{q} q^{\left(\begin{array}{c}
l-k \\
2
\end{array}\right)} x^{k} \mathcal{S}_{l-k, q^{-1}}(-1)\right) \mathscr{D}_{q, y} y^{n-l} \\
& =\sum_{l=0}^{n}\left[\begin{array}{l}
n \\
l
\end{array}\right]_{q}[n-l]_{q}\left(\sum_{k=0}^{l}\left[\begin{array}{l}
l \\
k
\end{array}\right]_{q} q^{\left.\left(\begin{array}{c}
l-k \\
2
\end{array}\right) x^{k} \mathcal{S}_{l-k, q^{-1}}(-1)\right) y^{n-l-1} .}\right.
\end{aligned}
$$

Comparing with Theorem 8 and result of the equation above, we can find the required result.

(ii) We omit a proof of (ii) due to its similarity to (i).

Theorem 11. Let $0<q<1$ and $q \in \mathbb{R}$. Then, we have

$$
\mathscr{D}_{q, y} \mathcal{S}_{n, q}(x, y)=\frac{\mathcal{S}_{n, q}(x, y)-\mathcal{S}_{n, q}(x, q y)}{(1-q) y} .
$$

Proof. We recall the definition of $q$-derivative such as

$$
\mathscr{D}_{q} f(x)=\frac{f(x)-f(q x)}{(1-q) x} \text {. }
$$

Applying $q$-derivative for $q$-sigmoid polynomials about parameter $y$, we obtain

$$
\begin{aligned}
\mathscr{D}_{q, y} \sum_{n=0}^{\infty} \mathcal{S}_{n, q}(x, y) \frac{t^{n}}{[n]_{q} !} & =\frac{1}{(1-q) y}\left(\frac{e_{q}(t x) e_{q}(t y)}{e_{q}(-t)+1}-\frac{e_{q}(t x) e_{q}(t q y)}{e_{q}(-t)+1}\right) \\
& =\frac{1}{(1-q) y}\left(\sum_{n=0}^{\infty} \mathcal{S}_{n, q}(x, y) \frac{t^{n}}{[n]_{q} !}-\sum_{n=0}^{\infty} \mathcal{S}_{n, q}(x, q y) \frac{t^{n}}{[n]_{q} !}\right) .
\end{aligned}
$$

Hence, we finish a proof of Theorem 10 .

Corollary 4. From Theorem 11, one holds

$$
\mathscr{D}_{q, x} \mathcal{S}_{n, q}(x)=\frac{\mathcal{S}_{n, q}(x)-\mathcal{S}_{n, q}(q x)}{(1-q) x} .
$$

$$
\begin{aligned}
& \mathcal{S}_{0, q}=\frac{1}{2} \\
& \mathcal{S}_{1, q}=\frac{1}{4} \\
& \mathcal{S}_{2, q}=-\frac{1}{8 q}(-1+q)(2+q), \\
& \mathcal{S}_{3, q}=\frac{1}{16 q^{2}}(1+q)\left(4-4 q-3 q^{3}+q^{4}+q^{5}\right), \\
& \mathcal{S}_{4, q}=-\frac{1}{32 q^{6}}\left(q^{4}+q^{3}-q-1\right)(8+q(-8+q(8+q(-8+(q-4+q(-2+(-1+q) q(3+q))))))) .
\end{aligned}
$$

\section{The Observation of Scattering Zeros of the $q$-Sigmoid Polynomials}

In this section, we try to find approximate roots of sigmoid polynomials combined with $q$-numbers and visualize them to confirm their properties. We use Mathematica to understand the values of the approximate roots and the structure piled up and make some assumptions based on this.

First, some $q$-sigmoid polynomials can be confirmed as follows: 


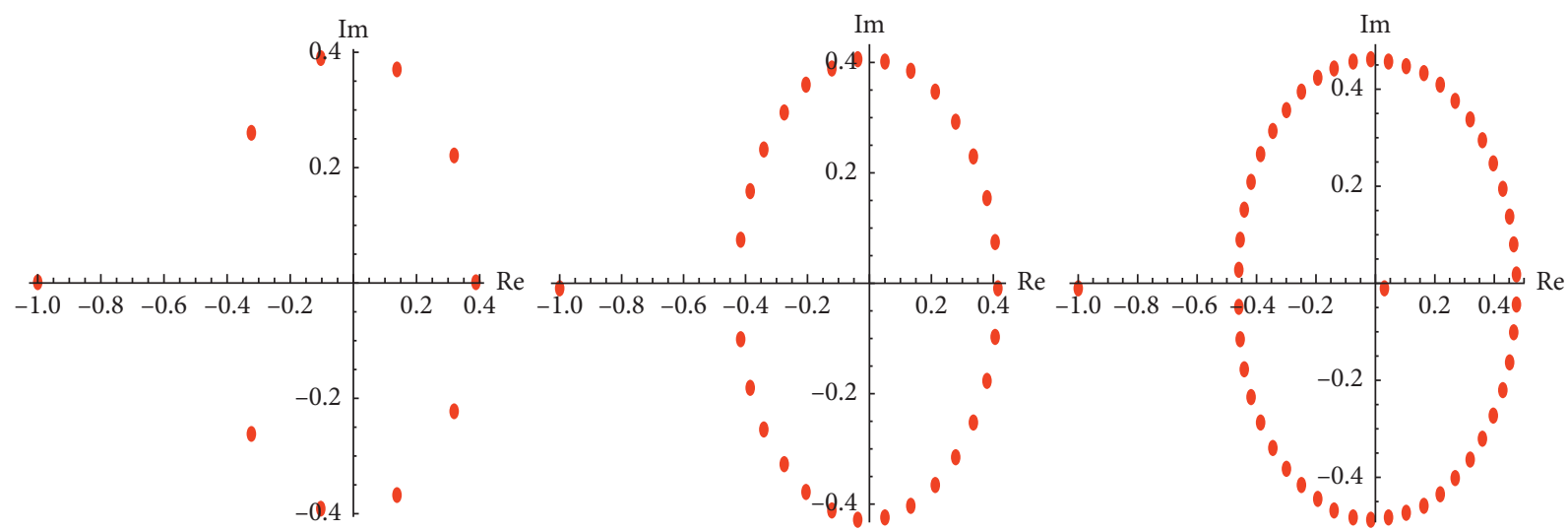

Figure 1: Zeros of $\mathcal{S}_{n, 0.1}(x)$ for $n=10,30,50$.

Furthermore, here are several $q$-sigmoid polynomials as follows:

$$
\begin{aligned}
& \mathcal{S}_{0, q}(x)=\frac{1}{2}, \\
& \mathcal{S}_{1, q}(x)=\frac{1}{4}(1+2 x), \\
& \mathcal{S}_{2, q}(x)=\frac{1}{8}\left(-1+q+2(1+q) x+4 x^{2}\right), \\
& \mathcal{S}_{3, q}(x)=\frac{1}{16}\left(1+q(-2+(-2+q) q)-2 x+2 q^{3} x+4\left(1+q+q^{2}\right) x^{2}+8 x^{3}\right), \\
& \mathcal{S}_{4, q}(x)=\frac{1}{32}\left(-1+3 q+3 q^{2}-3 q^{4}-3 q^{5}+q^{6}+2(1+q)^{2}(1+(-3+q) q)\left(1+q^{2}\right) x\right. \\
& \left.\quad+4\left(-1+q^{2}\left(-1+q+q^{3}\right)\right) x^{2}+8(1+q)\left(1+q^{2}\right) x^{3}+16 x^{4}\right) .
\end{aligned}
$$

Based on this polynomial, through the program we try to examine the location of the polynomial roots when the $q$-number condition is different. Here we assume that the position of the approximate roots will be the point that constitutes the circle as the order of the polynomial increases. The reason is because Bernoulli, Euler, and Genocchi polynomials, which combine actual $q$-numbers, often appear in such a way. First, Figure 1 shows the case when the $q$-number is fixed at 0.1 . The value of $n$ is set to the left (10), the middle (30), and the right (50). It can be seen that the feature here is always an approximation of -1.0 on the real axis. Next, as $n$ increases, the approximate roots form a circular shape.

Figure 2 shows the result when $n$ changes from 10,30 , to 50 when $q$ is fixed to 0.5 . The feature shown here appears from $n=30$. It can be seen that approximate values of $x$ are apart between -0.4 and -0.1 . In addition, when $n=50$, it can be seen that a more approximate interval is widened. In the case of $n=50$, there exists a symmetrical root with respect to the real axis.

Table 1 shows the actually calculated approximate values for $q=0.5$ and $n=50$. Based on Table 1 , it can be seen that the approximate value of the root still remains circular, but it does not exist since the circular form is widened when the value of $x$ is close to -0.3 . The calculation done by using Mathematica shows that the radius is 0.5 , the center is the origin, and the angle of incidence is $0.25834^{\circ}$. Here, we can assume that if the $q$ goes to 1 due to this interval, symmetric approximate roots will emerge.

In Figure 3, when $q$ is fixed at 0.9 and $n$ changes from left to right, changes were given to $10,30,50$.

Figure 3 at $n=50$ shows symmetry, which is very similar to the sigmoid polynomial. If we choose a $q$-number close to 1 , it will be almost similar to the approximate figure of the sigmoid polynomial. Here, the axis of symmetry except real roots is $x \approx-0.25$ on the real axis and a spreading can be confirmed. It can be seen that the shape at this time is an elliptical shape, and the angle at which it is opened is $0.39404^{\circ}$.

Based on these results, we can confirm the accumulation structure of root in 3 dimensions as shown in Figure 4. It can be confirmed that the structure of root is different according to the change of $q$-number and the 


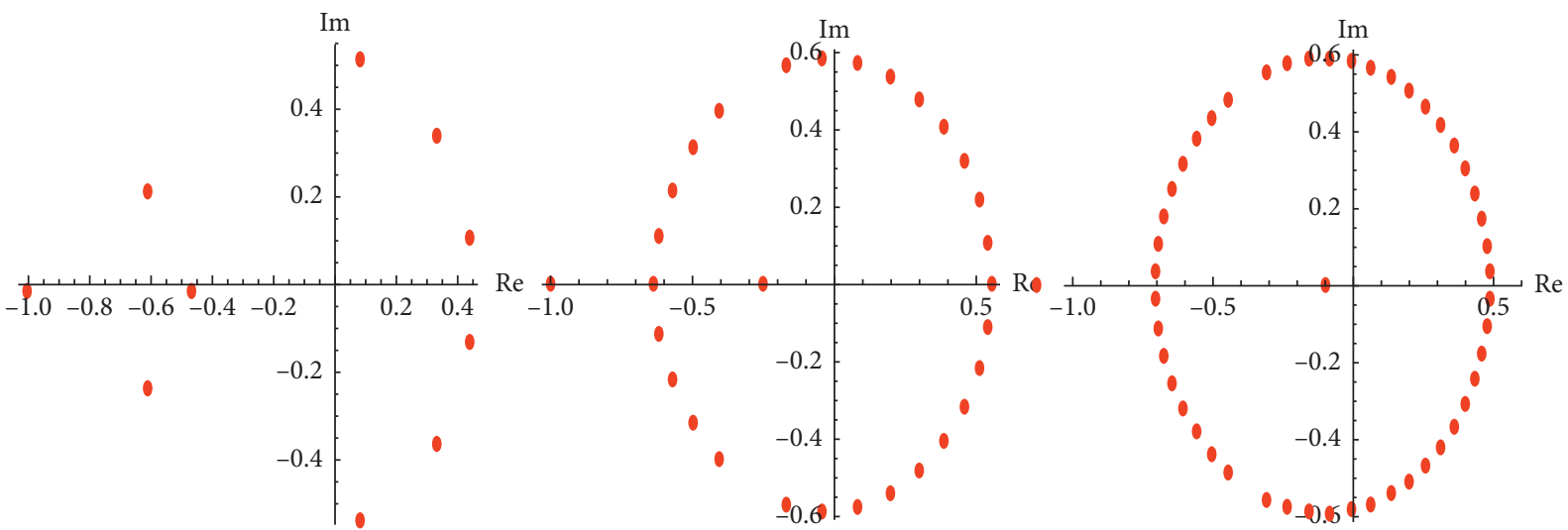

Figure 2: Zeros of $\mathcal{S}_{n, 0.5}(x)$ for $n=10,30,50$.

TABLE 1: Approximate zeros of $\mathcal{S}_{50,0.5}(x)$.

\begin{tabular}{cc}
\hline$q=0.5$ and $n=50$ \\
\hline$-1,-0.620721-0.0361286 i,-0.620721+0.0361286 i$ \\
$x$ & $-0.60958-0.108614 i,-0.60958+0.108614 i,-0.588669-0.180732 i$ \\
$-0.588669+0.180732 i,-0.558909-0.250923 i,-0.558909+0.250923 i$ \\
$-0.520715-0.31767 i,-0.520715+0.31767 i,-0.474449-0.379636 i$ \\
$-0.474449+0.379636 i,-0.420612-0.435565 i,-0.420612+0.435565 i$ \\
$-0.359937-0.484249 i,-0.359937+0.484249 i,-0.222503-0.555698 i$ \\
$-0.222503+0.555698 i,-0.148551-0.577093 i-0.148551+0.577093 i$ \\
$-0.0730754-0.588612 i,-0.0730754+0.588612 i,-0.0137537$ \\
$0.00256565-0.590362 i, 0.00256565+0.590362 i, 0.0771461-0.58261 i$ \\
$0.0771461+0.58261 i, 0.14955-0.565738 i, 0.14955+0.565738 i$ \\
$0.218754-0.540216 i, 0.218754+0.540216 i, 0.283816-0.506599 i$ \\
$0.283816+0.506599 i, 0.343876-0.46552 i, 0.343876+0.46552 i$ \\
$0.398157-0.417686 i, 0.398157+0.417686 i, 0.445974-0.363867 i$ \\
$0.445974+0.363867 i, 0.486731-0.304893 i, 0.486731+0.304893 i$ \\
$0.519934-0.241641 i, 0.519934+0.241641 i, 0.545183-0.175026 i$ \\
$0.545183+0.175026 i, 0.562181-0.105989 i, 0.562181+0.105989 i$
\end{tabular}

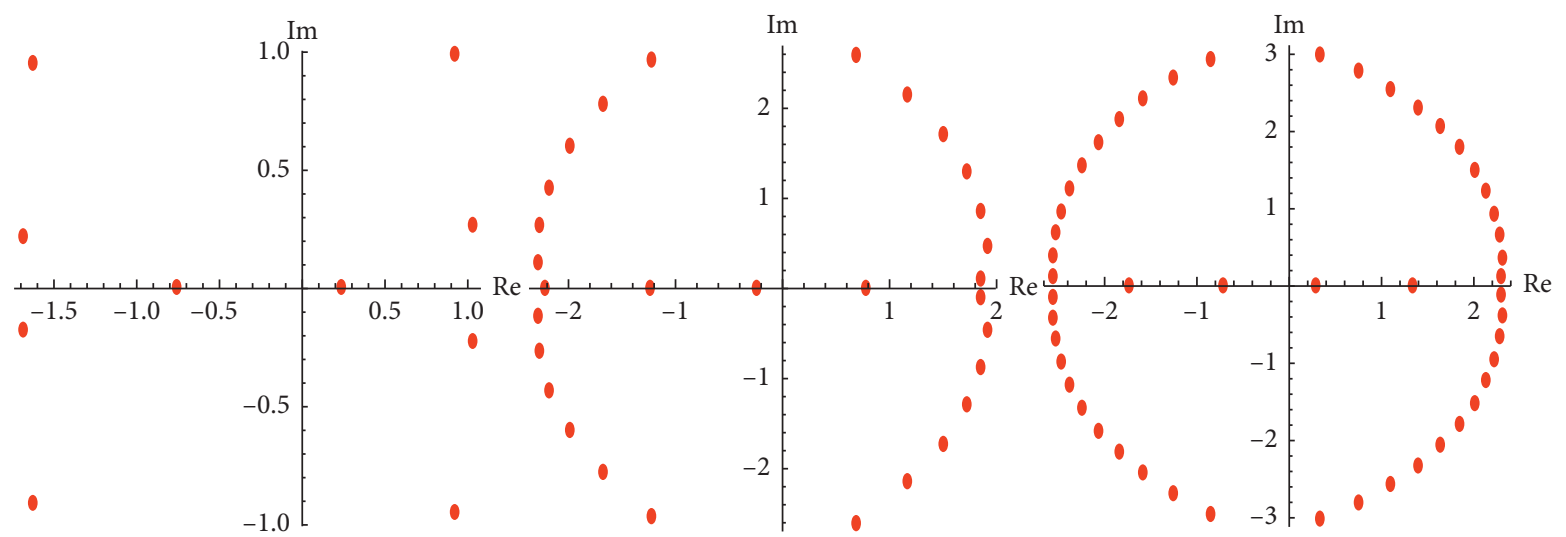

Figure 3: Zeros of $\mathcal{S}_{n, 0.9}(x)$ for $n=10,30,50$.

change of the value of $n$. Since we can see that the structure on the right side is almost similar to the structure of a general sigmoid polynomial, the form of sigmoid polynomial combining $q$-numbers can be thought of as the left figure and the middle figure. Therefore, we can think of the following conjecture.

\section{Conjecture 1}

(1) When $q$ is close to 0 and $n \geq 50$, the distribution of the roots is close to the circle.

(2) When $q=50$ with $n \geq 50$, the distribution of the roots is spread out. 


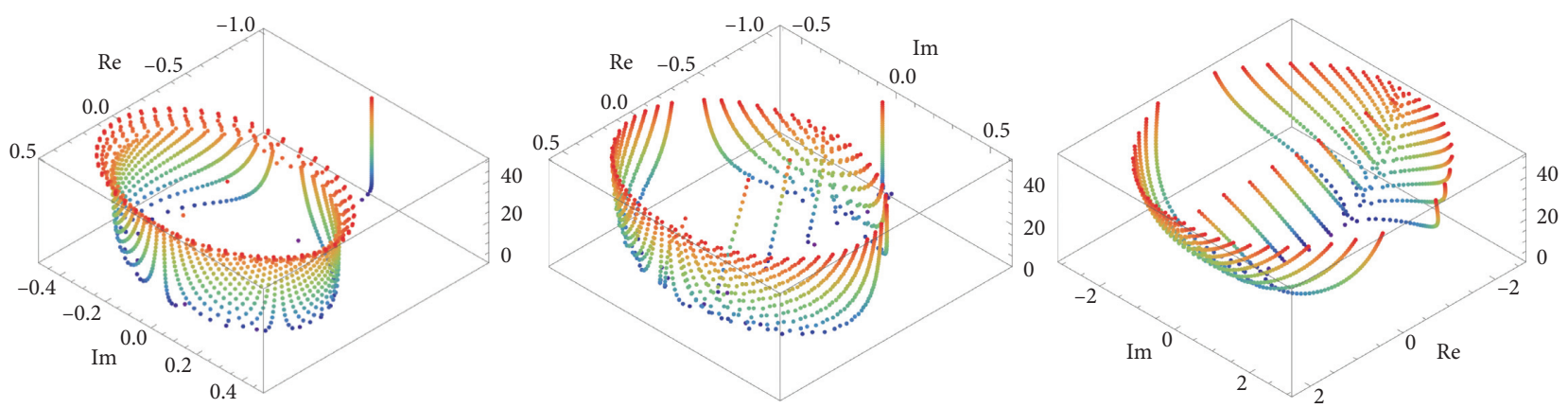

Figure 4: Zeros of $\mathcal{S}_{n, q}(x)$ for $q=0.1,0.5,0.9,1 \leq n \leq 50$.

(3) When $q \leq 0.5$ with $n \geq 10$, one of the approximate roots has a certain real root.

\section{Conclusion}

In this paper, approximate roots are obtained by programming based on the theorems in Section 2. By looking for the guesses in Section 3, we were able to visually confirm the properties of the $q$-number. I think more research is needed regarding what happens to the distribution of the approximate roots according to the angles.

\section{Data Availability}

The data used to support the findings of this study are available from the corresponding author upon request.

\section{Conflicts of Interest}

The author declares that there are no conflicts of interest regarding the publication of this paper.

\section{Acknowledgments}

This research was supported by Basic Science Research Program through the National Research Foundation of Korea (NRF) funded by the Ministry of Science, ICT and Future Planning (no. 2017R1E1A1A03070483).

\section{References}

[1] G. E. Andrews, R. Askey, and R. Roy, Special Functions, Cambridge Press, Cambridge, UK, 1999.

[2] M. Arik, E. Demircan, T. Turgut, L. Ekinci, and M. Mungan, "Fibonacci oscillators," Zeitschrift für Physik C Particles and Fields, vol. 55, no. 1, pp. 89-95, 1992.

[3] G. Bangerezako, An Introduction to q-difference Equations, preprint, Bujumbura, Burundi, 2008.

[4] R. D. Carmichael, "The general theory of linear q-difference equations," American Journal of Mathematics, vol. 34, no. 2, pp. 147-168, 1912.

[5] H. Exton, q-Hypergeometric Functions and Applications, Ellis Horwood, Chichester, UK, 1983.

[6] F. H. Jackson, "q-Difference Equations," American Journal of Mathematics, vol. 32, no. 4, pp. 305-314, 1910.
[7] H. F. Jackson, "On $q$-functions and a certain difference operator," Transactions of the Royal Society of Edinburgh, vol. 46, pp. 253-281, 1909.

[8] V. Kac and P. Cheung, Quantum Calculus, Springer-Verlag, Berlin, Germany, 2002.

[9] T. E. Mason, "On properties of the solutions of linear q-difference equations with entire function coefficients," American Journal of Mathematics, vol. 37, no. 4, pp. 439-444, 1915.

[10] W. J. Trjitzinsky, "Analytic theory of linear q-difference equations," Acta Mathematica, vol. 69, pp. 1-38, 1933.

[11] R. Ayoub, "Euler and the zeta function," The American Mathematical Monthly, vol. 81, no. 10, pp. 1067-1086, 1974.

[12] L. Comtet, Advanced Combinatorics, Reidel, Dordrecht, The Netherlands, 1974.

[13] G.-S. Cheon, "A note on the Bernoulli and Euler polynomials," Applied Mathematics Letters, vol. 16, no. 3, pp. 365$368,2003$.

[14] S. Endre and M. David, An Introduction to Numerical Analysis, Cambridge University Press, Cambridge, UK, 2003.

[15] J. Mira and F. Sandoval, "From natural to artificial neural computation," in Proceedings of the International Workshop on Artificial Neural Networks, pp. 195-201, Malaga-Torremolinos, Spain, June 1995.

[16] J. Han, R. S. Wilson, and S. E. Leurgans, "Sigmoidal mixed models for longitudinal data," Statistical Methods in Medical Research, vol. 27, no. 3, pp. 863-875, 2018.

[17] Y. Ito, "Representation of functions by superpositions of a step or sigmoid function and their applications to neural network theory," Neural Networks, vol. 4, no. 3, pp. 385-394, 1991.

[18] H. K. Kwan, "Simple sigmoid-like activation function suitable for digital hardware implementation," Electronics Letters, vol. 28, 1992.

[19] M. Kim, Y. Song, S. Wang, Y. Xia, and X. Jiang, "Secure logistic regression based on homomorphic encryption: design and evaluation," JMIR Medical Informatics, vol. 6, no. 2, p. e19, 2018.

[20] D. E. Knuth and T. J. Buckholtz, "Computation of tangent, Euler, and Bernoulli numbers," Mathematics of Computation, vol. 21, no. 100 , p. 663, 1967.

[21] C. T. Kelley, Solving Nonlinear Equations with Newton's Method, SIAM, Philadelphia, PA, USA, 2003.

[22] J. Konvalina, "A unified interpretation of the binomial coefficients, the stirling numbers, and the Gaussian coefficients," The American Mathematical Monthly, vol. 107, no. 10, pp. 901-910, 2000.

[23] J. Y. Kang, "Some relationships between sigmoid polynomials and other polynomials," Journal of Applied and Pure Mathematics, vol. 1, no. 1-2, pp. 57-67, 2019. 
[24] N. I. Mahmudov, "A new class of generalized bernoulli polynomials and euler polynomials," p. 31, 2012, http://arxiv. org/abs/1201.6633v1.

[25] C. S. Ryoo, "Differential equations associated with tangent numbers," Journal of Applied Mathematics \& Informatics, vol. 34, no. 5_6, pp. 487-494, 2016. 